\title{
Growth Differentiation Factor-15 and Syndecan-1 Are Potential Biomarkers of Cardiac and Renal Involvement in Classical Fabry Disease under Enzyme Replacement Therapy
}

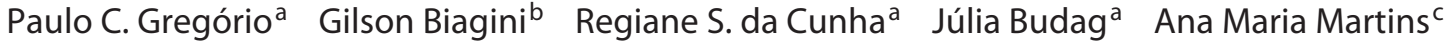 \\ Lara Valiño Rivas $^{d, e}$ Elberth M. Schiefer ${ }^{f}$ Maria Dolores Sánchez-Niño ${ }^{e, g} \quad$ Alberto Ortiz $^{d, e}$
} Andréa E. M. Stinghen ${ }^{\text {a }}$ Fellype C. Barreto ${ }^{\text {h }}$

aBasic Pathology Department, Experimental Nephrology Laboratory, Universidade Federal do Paraná, Curitiba, Brazil; bepartment of Internal Medicine, Postgraduate Program in Internal Medicine and Health Sciences, Universidade Federal do Paraná, Curitiba, Brazil; 'Peadiatrics, Universidade Federal de São Paulo, Unifesp, São Paulo, Brazil; dNephrology and Hypertension, Hospital Universitario Fundación Jiménez Díaz, Madrid, Spain; elIS-Fundación

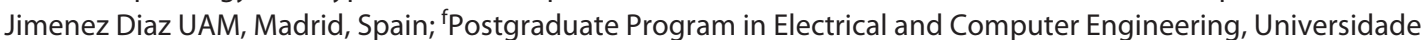
Tecnológica Federal do Paraná, Curitiba, Brazil; ${ }^{9}$ Department of Pharmacology, School of Medicine, Universidad Autónoma de Madrid, Madrid, Spain; ${ }^{\text {h}}$ Department of Internal Medicine, Service of Nephrology, Universidade Federal do Paraná, Curitiba, Brazil

\section{Highlights}

- Fabry disease patients are mainly monitored by traditional biomarkers of target organ injury, such as serum creatinine and proteinuria, which provide no information about inflammation and endothelial damage, both considered the main pathophysiologic pathways that lead to Fabry-related long-term complications.

- Growth differentiation factor-15 (GDF-15) and syndecan-1 were associated with evidence of heart and kidney injury in classical Fabry patients on enzyme replacement therapy (ERT). Moreover, together with 3-nitrotyrosine, time on ERT, and arterial pressure, they may serve as important variables to identify the patients with both cardiac and renal involvement.

- The potential association of GDF-15 and syndecan-1 with residual risk and disease outcomes in Fabry patients provides a framework to incorporate novel biomarkers in the monitoring of these patients.

\section{Keywords}

Fabry disease · Enzyme replacement therapy · Biomarkers · Inflammation · Oxidative stress

Gilson Biagini (in memorian).

\section{Abstract}

Background and Aims: Inflammation and endothelial damage play a pivotal role in Fabry disease (FD) manifestations. In daily clinical practice, FD is mainly monitored by traditional biomarkers of target organ injury, such as serum creatinine and proteinuria, which provide no information about inflammation and endothelial damage. Materials and Methods: We investigated the serum levels of 3-nitrotyrosine (3-NT),
Karger@karger.com www.karger.com/kbr

Karger $\stackrel{\text { ' }}{5}$ GOPEN ACCESS
(C) 2022 The Author(s)

Published by S. Karger AG, Basel

This is an Open Access article licensed under the Creative Commons Attribution-NonCommercial-4.0 International License (CC BY-NC) (http://www.karger.com/Services/OpenAccessLicense), applicable to the online version of the article only. Usage and distribution for commercial purposes requires written permission.
Correspondence to:

Fellype Carvalho Barreto, fellype.barreto@ufpr.br 
an oxidative stress biomarker, and of growth differentiation factor-15 (GDF-15) and syndecan-1 in classical FD patients on enzyme replacement therapy (ERT) for at least 6 months and their relationship with Fabry-related cardiac and renal manifestations. Results: Fifty-two classical FD patients (37 females) on ERT for $62.0 \pm 27.5$ months were included in the study. The main clinical manifestations included nephropathy $(67.3 \%)$ and cardiomyopathy (21.1\%). Serum levels of 3-NT, syndecan-1, and GDF-15 were $33.3(4.8-111.1) \mathrm{nmol} /$ $\mathrm{mL}, 55.7$ (38.8-74.9) $\mathrm{ng} / \mathrm{mL}$, and 541.8 (392.2-784.4) pg/mL, respectively. There was a direct correlation between interventricular septal thickness and serum GDF-15 ( $r=0.59 ; p<$ $0.001)$ and syndecan- $1(r=0.30, p=0.04)$. Among kidney parameters, there was a significant correlation between estimated glomerular filtration rate and GDF-15 $(r=-0.61 ; p<$ 0.001 ), as well as between $24 \mathrm{~h}$ proteinuria and syndecan-1 $(r=0.28 ; p=0.04)$. Serum GDF-15 levels were significantly higher in patients with cardiomyopathy $(p=0.03)$ as well in those with both nephropathy and cardiomyopathy $(p=0.02)$ than in patients without these comorbidities. Serum GDF-15 levels were also significantly higher in patients who started ERT at an older age ( $\geq 40$ years). In multivariate analysis, syndecan-1, 3-NT, GDF-15, time on ERT, and arterial pressure differentiated Fabry patients with both cardiac and renal involvement from those without these manifestations. Conclusions: GDF-15 and syndecan-1 were associated with parameters of cardiac and renal involvement in classic FD patients on ERT. Their potential association with residual risk and disease outcomes should be investigated.

(c) 2022 The Author(s).

Published by S. Karger AG, Basel

\section{Introduction}

Fabry disease (FD) is a rare $\mathrm{X}$-linked disorder characterized by partial or complete deficiency of the enzyme a-galactosidase A due to mutations in the GLA gene, which leads to lysosomal accumulation of glycosphingolipids, mainly globotriaosylceramide (Gb3) and its deacylated metabolite, globotriaosylsphingosine (lyso-Gb3) $[1,2]$. The progressive accumulation of these compounds over time causes cellular dysfunction and subsequent damage to vital organs such as the heart, the brain, and the kidneys [1-3].

Two phenotypes of FD are recognized. In the classical phenotype, manifestations, such as acroparesthesias, angiokeratomas, hypohidrosis, and gastrointestinal symptoms, begin during childhood or adolescence [2]. Adults may develop major organ involvement, such as protein- uric chronic kidney disease (CKD), left ventricular hypertrophy $(\mathrm{LVH})$, and cerebrovascular disease and may suffer from end-stage kidney disease, cardiac and cerebrovascular events, and, eventually, premature death $[4,5]$. In the later-onset phenotype, manifestations are primarily limited to the heart or to the kidneys, and the early manifestations of the classical phenotype may be either absent or appear later. Importantly, due to random Xinactivation, FD manifestations in heterozygous females are variable, ranging from asymptomatic to severity similar to males with the same mutation $[6,7]$.

Vasculopathy has been identified as the driving force for the life-threatening complications of FD. Gb3 accumulation may cause endothelial dysfunction by decreasing the expression of endothelial nitric oxide synthase and the bioavailability of nitric oxide $[8,9]$. Gb3 may also increase oxidative stress and upregulate the expression of plasminogen activator inhibitor and cell adhesion molecules, such as vascular cell adhesion molecule-1, P-selectin, and E-selectin $[10,11]$. Additionally, lyso-Gb3 may activate pro-inflammatory and profibrotic pathways that further amplify cell and target organ injury $[12,13]$. Thus, endothelial dysfunction and inflammation may underlie the long-term complications in FD.

Enzyme replacement therapy (ERT) is available in two formulations, agalsidase- $\alpha(0.2 \mathrm{mg} / \mathrm{kg} / \mathrm{e} . \mathrm{o.w})$ and agalsidase- $\beta$ (1.0 mg/kg/e.o.w). ERT, particularly at higher doses, promotes short- and long-term clearance of Gb3 deposits in podocytes and in kidney endothelial cells, the heart, and the skin [14-16]. Importantly, timely initiation of ERT has been associated with slower progression of renal function decline, $\mathrm{LVH}$, and white matter lesions [17-22]. In the absence of timely treatment, patients may develop severe clinical complications, even while on ERT, when ERT was initiated at an older age, evidencing a possible residual risk [23]. Currently, the availability of serum biomarkers of tissue injury is limited to proteinuria in the routine clinical setting, with additional biomarkers, such as serum creatinine, N-terminal-pro-BNP, and high-sensitivity troponin being used to detect organ dysfunction [24]. In the present study, we have evaluated serum biomarkers related to oxidative stress (3-nitrotyrosine [3-NT]), endothelial dysfunction (syndecan-1, a biomarker of endothelial dysfunction and glycocalyx damage), and kidney injury/kidney and cardiovascular outcomes (growth differentiation factor-15 [GDF-15]) in a cohort of classical FD patients receiving ERT to identify possible biomarkers of organ damage. 


\section{Methodology}

Study Design and Assessments

This cross-sectional and observational study included classical FD patients that had been on regular follow-up and under ERT for at least 6 months in two centers in Curitiba and in Tapejara, Brazil. The classical phenotype of FD was confirmed by genotyping and by clinical manifestations. Patients with other recognized causes of CKD were excluded. Demographic, clinical, and laboratory data were collected from medical charts. ERT was initiated for symptomatic FD or for the presence of subclinical manifestations of major organ involvement. Criteria for ERT initiation included neuropathic pain, estimated glomerular filtration rate (eGFR) $<90 \mathrm{~mL} / \mathrm{min} / 1.73 \mathrm{~m}^{2}$, albuminuria $>30 \mathrm{mg} / \mathrm{g}, \mathrm{LVH}$, arrhythmia, transient ischemic attack, stroke, and disabling gastrointestinal symptoms, among others [25]. eGFR was calculated from serum creatinine using the CKD epidemiology collaboration equation [26]. Glomerular hyperfiltration was defined as an eGFR $>130 \mathrm{~mL} / \mathrm{min} / 1.73 \mathrm{~m}^{2}$, corrected for age (>40 years old: $-1 \mathrm{~mL} /$ $\mathrm{min} / 1.73 \mathrm{~m}^{2} /$ year) [27], as previously proposed [28]. Proteinuria was measured in 24-h urine samples. Fabry cardiomyopathy was defined as the presence of electrocardiogram abnormalities, $\mathrm{LVH}$, or interventricular septal hypertrophy, as measured by bidimensional transthoracic echocardiography and defined as LV wall thickness $>12 \mathrm{~mm}$ in males and $>11 \mathrm{~mm}$ in females, and as interventricular septum $>11 \mathrm{~mm}$, respectively. Fabry nephropathy was defined by low eGFR $\left(<90 \mathrm{~mL} / \mathrm{min} / 1.73 \mathrm{~m}^{2}\right)$ or abnormal levels of albuminuria ( $>30 \mathrm{mg} / \mathrm{g}$ ) or proteinuria $(>150 \mathrm{mg} / 24 \mathrm{~h})$. Patients were categorized into CKD stages as proposed by Kidney Disease: Improving Global Outcomes Clinical Practice Guideline for the Evaluation and Management of CKD [29]. Based on prior knowledge of the histological features of FD, they were assumed to have at least glycolipid deposits in kidney cells as for other classic FD patients and, thus, were considered to fulfill the Kidney Disease: Improving Global Outcomes criterion for CKD of "Abnormalities detected by histology" even in the absence of kidney biopsy for individual persons. A similar criterion was previously used in the FD field [30]. Severe clinical events were defined as need for renal replacement therapy, such as dialysis or kidney transplantation, stroke or transient ischemic attack, acute myocardial infarction, or implantation of pacemaker or of implantable cardiovascular defibrillator.

This study protocol was approved by the Ethics Committee of Universidade Federal do Paraná (Curitiba, Brazil) under registration number CEP/UFPR n 2.209.616. All patients provided signed informed consent.

\section{Measurement of Syndecan-1, 3-NT, and GDF-15}

Serum samples were collected from patients in treatment prior to ERT infusion and stored at $-80^{\circ} \mathrm{C}$ until analysis. Serum concentrations of syndecan-1 (Abcam, Cambridge, UK), 3-NT (Abcam, Cambridge, UK), and GDF-15 (RD systems, Minneapolis, MN, USA) were measured using an enzyme-linked immunosorbent assay. Samples were analyzed simultaneously under standardized experimental conditions. All measurements were performed in duplicate. The assay ranges for syndecan-1, 3-NT, and GDF-15 were $0-256 \mathrm{ng} / \mathrm{mL}, 0-8,000 \mathrm{nmol} / \mathrm{mL}$, and $0-1,500 \mathrm{pg} /$ $\mathrm{mL}$, respectively.

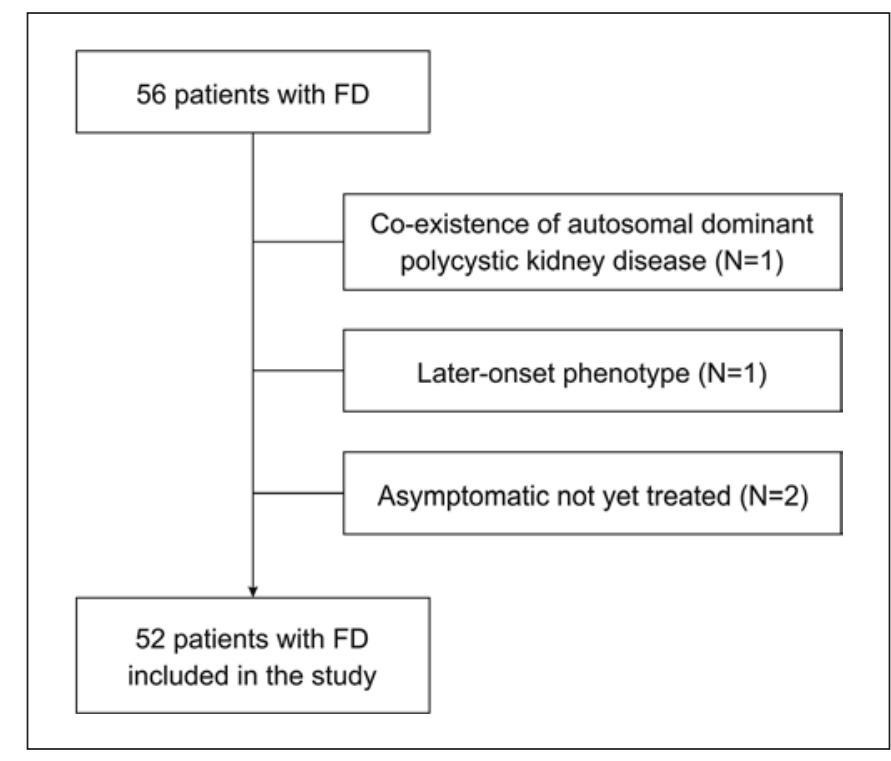

Fig. 1. Study flow diagram. Out of fifty-six $(N=56)$ Fabry patients, 4 patients $(N=4)$ were not included in study due to coexistence of autosomal dominant polycystic kidney disease $(N=1)$, later-onset phenotype $(N=1)$, or being asymptomatic not yet treated $(N=2)$.

\section{Statistical Analyses}

Statistical analyses were performed using the statistical packages JMP (version 8.0; SAS Institute Inc., Cary, NC, USA) and SigmaStat (version 3.5; Systat software Inc., Erkrath, Germany). Comparisons between groups were performed using Student's $t$ test or analysis of variance for paired data, and using Mann-Whitney and analysis of variance on Rank's for unpaired data. To investigate the association of later start of ERT with biochemical and clinical abnormalities, patients were divided into two groups according to their age at ERT initiation, with a cutoff point at 40 years, which represents the mean age at ERT initiation for large ( $n>1,000$ patients) series and has been used previously in the literature [31]. To investigate differences on the serum levels of the novel biomarkers according to renal damage, patients were also grouped according to the CKD stage and to the tertile of eGFR. Pearson's or Spearman's correlation coefficients were used to assess the relationship between syndecan-1,3-NT, and GDF-15 and clinical and biochemical variables. Partial least squares (PLS) regressions were calculated using OriginPro 2016. PLSs were then used to further evaluate the relationship among clinical and laboratory variables and Fabry cardiomyopathy and nephropathy through a variable importance plot (VIP). Variables considered of clinical importance, such as systolic and diastolic blood pressures, systemic arterial hypertension, time on ERT, gender, and age as well as the novel biomarkers (GDF-15, syndecan-1, and 3-NT) were included in the model. Statistical differences $(p<0.05)$ among groups of patients were evaluated by confidence ellipses in the PLS score plots. Variables with VIP higher than 0.8 were considered moderately or highly influential to the separation of groups [28]. Values were expressed as mean \pm standard deviation or as median (percentiles 25-75). $p<0.05$ was considered statistically significant. 
Table 1. Demographic and laboratory characteristics of the studied population $(N=52)$

\begin{tabular}{|c|c|c|c|}
\hline & \multirow[t]{2}{*}{ Cohort $(N=52)$} & \multicolumn{2}{|l|}{ Age at ERT initiation } \\
\hline & & $<40$ years $(N=33)$ & $\geq 40$ years $(N=19)$ \\
\hline Age at diagnosis, years & $31.9 \pm 15.2$ & $21.7 \pm 7.6$ & $48.6 \pm 8.0^{*}$ \\
\hline Age at initiation of ERT, years & $34.1 \pm 15.8$ & $24.1 \pm 8.1$ & $51.7 \pm 9.0^{*}$ \\
\hline Female, $N(\%)$ & $37(71)$ & $21(63.6)$ & $16(84.2)$ \\
\hline Caucasian, $N(\%)$ & $50(96)$ & $32(97.0)$ & $18(94.7)$ \\
\hline \multicolumn{4}{|l|}{ Genotyping, $N(\%)$} \\
\hline p.y365x & $36(69.2)$ & $21(63.6)$ & 15 (78.9) \\
\hline p.w47x & $9(17.3)$ & $6(18.2)$ & $3(15.8)$ \\
\hline Other (c.352c>t; c.332delG; p.w204x) & $7(13.5)$ & $6(18.2)$ & $1(5.3)$ \\
\hline RAS blockade, $N(\%)$ & $26(50)$ & $18(54)$ & $8(42)$ \\
\hline IVST, $N(\%)$ & $47(90.3)$ & $29(55.7)$ & $18(34.6)$ \\
\hline IVST, mm & $11.2 \pm 3.5$ & $9.5 \pm 1.6$ & $14.0 \pm 3.8^{* * * *}$ \\
\hline \multicolumn{4}{|l|}{ FD manifestations, $N(\%)$} \\
\hline Cardiomyopathy & $11(21.1)$ & $5(15.2)$ & $6(31.6)$ \\
\hline Nephropathy & $35(67.3)$ & $24(72.7)$ & $11(57.9)$ \\
\hline Cardiomyopathy and nephropathy & $5(9.6)$ & $1(3.0)$ & $4(21.1)$ \\
\hline Time from diagnosis until ERT initiation, months & $36.0 \pm 25.8$ & $34.7 \pm 24.5$ & $36.9 \pm 28.6$ \\
\hline Time on ERT, months & $62.0 \pm 27.5$ & $60.4 \pm 28.1$ & $64.8 \pm 27.2$ \\
\hline \multicolumn{4}{|l|}{ CKD stage, $N(\%)$} \\
\hline 1 & $39(75)$ & $29(88)$ & $10(52.6)^{* * *}$ \\
\hline 2 & $8(15.4)$ & $2(6)$ & $6(31.6)^{* *}$ \\
\hline $3 a$ & $5(9.6)$ & $2(6)$ & $3(15.8)$ \\
\hline \multicolumn{4}{|l|}{ Biochemical parameters } \\
\hline Serum creatinine, $\mathrm{mg} / \mathrm{dL}$ & $0.80 \pm 0.004$ & $0.75 \pm 0.25$ & $0.87 \pm 0.21^{* *}$ \\
\hline $\mathrm{eGFR}, \mathrm{mL} / \mathrm{min} / 1.73 \mathrm{~m}^{2}$ & $104.7 \pm 26.8$ & $117.6 \pm 24.2$ & $84.6 \pm 18.4^{*}$ \\
\hline Proteinuria, mg/24 h & $298.6 \pm 387.7$ & $324.5 \pm 385.0$ & $251.7 \pm 388.3$ \\
\hline \multicolumn{4}{|l|}{ Serum inflammatory biomarkers ${ }^{a}$} \\
\hline 3-NT, $\mathrm{nmol} / \mathrm{mL}$ & $33.3(4.8-111.1)$ & $23.7(4.6-90.0)$ & $38.8(5.8-111.1)$ \\
\hline Syndecan-1, ng/mL & $55.1(38.8-74.9)$ & $62.0(35.8-83.3)$ & $47.2(39.6-64.4)$ \\
\hline GDF-15, pg/mL & $541.8(392.2-784.4)$ & $450.3(377.4-544.3)$ & $739.9(614.8-978.0)^{*}$ \\
\hline
\end{tabular}

Values as mean $\pm \mathrm{SD}$, median (percentiles 25-75), or percentage. RAS, renin angiotensin system; SD, standard deviation. ${ }^{a}$ Values in a control healthy population were: $38(57-236) \mathrm{nm} / \mathrm{mL}$ for 3-NT; 53 (39-261) ng/mL for syndecan-1; and 381 (298-450) pg/mL for GDF-15. * $p<0.0001,{ }^{* *} p<0.05,{ }^{* * *} p<0.01,{ }^{* * * *} p<0.0001$ versus $<40$ years.

\section{Results}

\section{Clinical and Laboratory Characteristics of the Study Population}

Fifty-six patients were selected to participate in this study. Four patients were excluded due to the following reasons: coexistence of autosomal dominant polycystic kidney disease $(N=1)$, later-onset phenotype $(N=1)$, and asymptomatic not yet treated $(N=2)$. A total of fifty-two patients were included in the study, most of them diagnosed through family screening (Fig. 1). The main clinical and laboratory characteristics of the study population are shown in Table 1. Patients were predominantly adults $(N=51)$ and females $(71 \%, N=37)$. There was one 16-year-old person. The mean ages at diagnosis and at initiation of ERT were $31.9 \pm 15.2$ and $34.1 \pm 15.8$ years, respectively. The most frequent GLA genetic variant was p.y365x $(69.2 \%, N=36)$. Renal and cardiac involvements were present in $67.3 \%(N=35)$ and in $21.1 \%(N=11)$ of patients, respectively. Concerning CKD stage, $75 \%$ of the patients were classified as stage G1, $15.4 \%$ as stage G2, and $9.6 \%$ as stage G3a. Nine patients (6 males; 3 females) had glomerular hyperfiltration at the time point of the study. All of them were younger than 40 years old (age: $21.4 \pm 3.7$ years) and have been on ERT for $62 \pm 15.8$ months. Six of them had clinical proteinuria (A3) and 3 patients had albuminuria (A2). So that all patients with glomerular hyperfiltration were considered as having re- 
Fig. 2. Matrix of correlation between parameters of target organ lesion, classic (proteinuria), and novel (GDF-15, syndecan-1, and 3-NT) biomarkers. IVST, interventricular septal thickness; Prot, $24 \mathrm{~h}$ proteinuria; SDC-1, syndecan-1. ${ }^{* *} p<$ $0.001 ;{ }^{*} p=0.01 ;{ }^{*} p=0.04$.

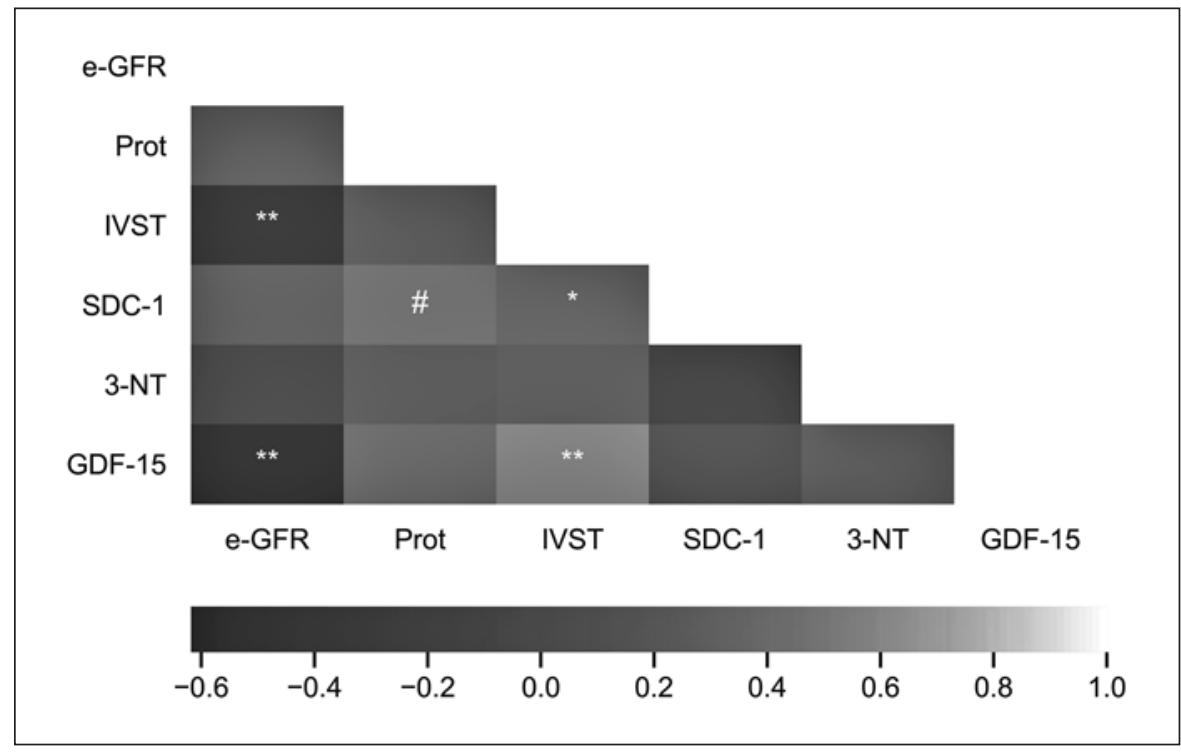

nal involvement (Fabry nephropathy) as they had some degree of albuminuria or proteinuria. Patients had been on ERT for $61.5 \pm 28.0$ months and had an overall followup of $96(72-108)$ months. No patient experienced any severe clinical event during this period.

\section{Age at Initiation of ERT and Biochemical and Clinical Parameters}

To investigate the association of later start of ERT with biochemical and clinical abnormalities, patients were divided into two groups according to their age at ERT initiation ( $<40$ vs. $\geq 40$-year-old, Table 1 ). As expected, eGFR was significantly lower whereas interventricular septal thickness (IVST) was significantly higher in patients who initiated ERT later. Regarding the novel biomarkers, serum GDF-15 levels were significantly higher in patients who started ERT at an older age. There was a significant association between age and GDF-15 ( $p<0.0001 ; r=$ 0.67 ). Syndecan- 1 and 3-NT were not significantly different between patients starting ERT at different ages.

Target Organ Injury and Classic (Proteinuria) or Novel (GDF-15, Syndecan-1, and 3-NT) Biomarkers

GDF-15 and syndecan-1 were correlated with features of target organ injury in classical FD patients on ERT. There was a significant correlation between eGFR and GDF-15 $(r=-0.62 ; p<0.001)$ as well as between 24 $\mathrm{h}$ proteinuria and syndecan- $1(r=0.28 ; p=0.04)$. Furthermore, there was a significant correlation between IVST and both GDF-15 $(r=0.59 ; p<0.001)$ and syndecan-1 $(r=0.30 ; p=0.04)$. There was also an inverse correlation between eGFR and IVST $(r=-0.45 ; p=$ 0.002) (Fig. 2).

When patients were grouped according to the CKD stage, serum levels of GDF-15 were significantly higher in CKD stage 3 in comparison with stage 1 (GDF-15: 1,071.4 $[520.4-1,532.7]$ vs. 475.1 [385.3-670.8] pg/mL; $p=0.02$ ). There was no difference neither between CKD stage 1 and 2 nor between CKD stage 2 and 3. Similarly, GDF-15 was only significantly higher in the lowest tertile of eGFR $\left(<95.80 \mathrm{~mL} / \mathrm{min} / 1.73 \mathrm{~m}^{2}\right)$ in comparison to the highest tertile eGFR $\left(\geq 121 \mathrm{~mL} / \mathrm{min} / 1.73 \mathrm{~m}^{2}\right)$; GDF-15: 767.8 $(539.4-1,062.2) \mathrm{pg} / \mathrm{mL}$ versus $387.2(314.5-460.4) \mathrm{pg} /$ $\mathrm{mL} ; p=0.001]$. There was no difference on the serum levels of 3-NT and syndecan-1 across the groups neither according to the CKD stages nor to the eGFR tertiles.

When patients were categorized according to the presence of target organ injury, serum GDF-15 levels were significantly higher in FD patients with cardiomyopathy, associated or not with nephropathy, than in patients without cardiomyopathy $(p<0.05)$ (Fig. 3a, c). GDF-15 serum levels were not different between patients with or without nephropathy (Fig. 3b). Syndecan-1 and 3-NT serum levels were not significantly different across groups (Fig. 3d-i).

\section{Time on ERT and GDF-15, Syndecan-1, and 3-NT}

Levels

There was a significant correlation between time on ERT and GDF-15 $(r=0.28 ; p=0.04)$. There was no correlation between time on ERT and both 3 -NT $(r=0.18$; $p=0.18)$ and syndecan- $1(r=-0.09 ; p=0.52)$ (Fig. 4). 


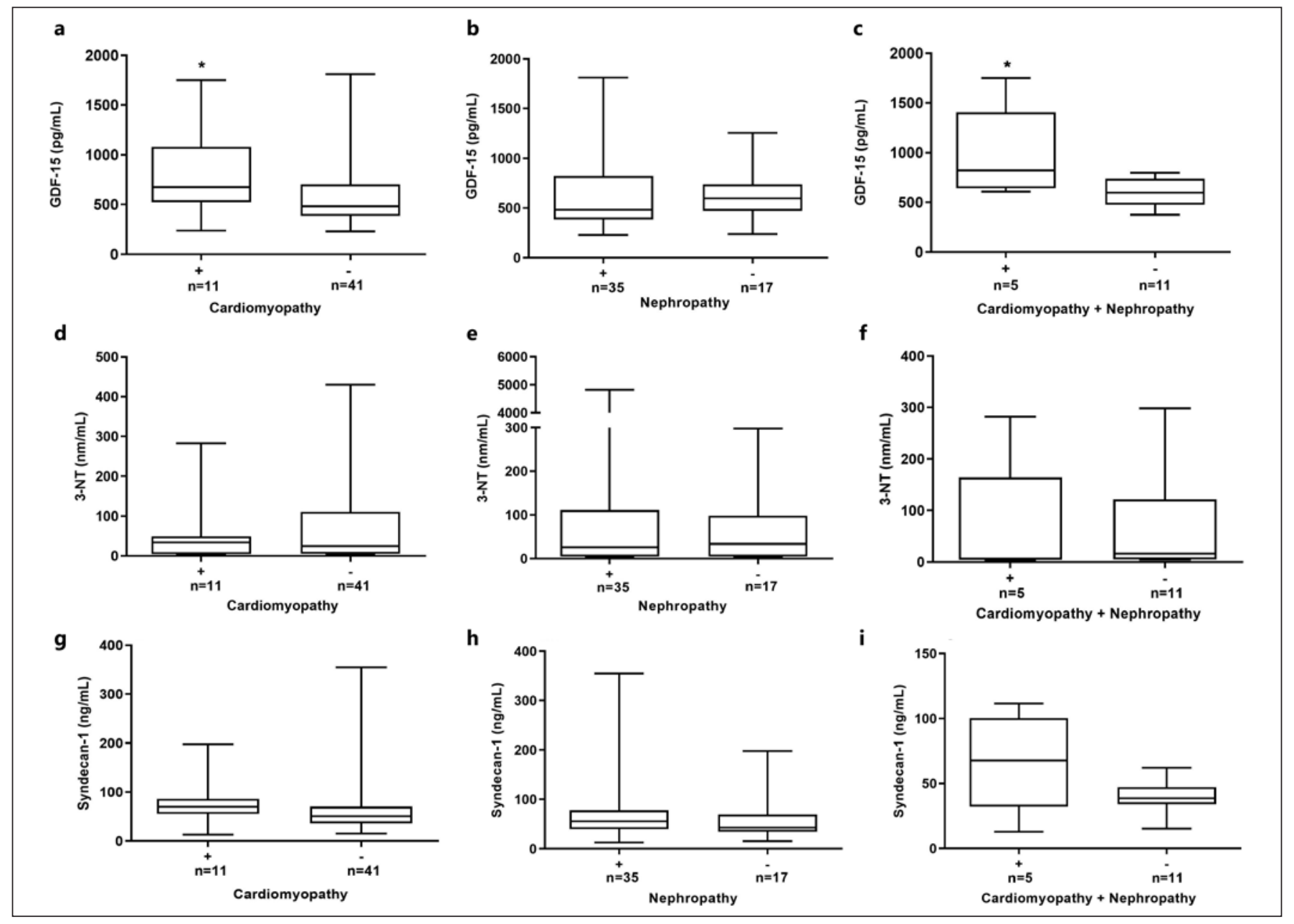

Fig. 3. Serum levels of GDF-15, 3-NT, and syndecan-1 in FD patients with or without cardiomyopathy and nephropathy. GDF-15 (a-c), 3-NT (d-f), and syndecan-1 levels $(\mathbf{g}-\mathbf{i})$ were measured by ELISA. Nephropathy was defined by low eGFR $(<90 \mathrm{~mL} / \mathrm{min} / 1.73$ $\mathrm{m}^{2}$ ) or abnormal levels of albuminuria/proteinuria $(>30 \mathrm{mg} / \mathrm{g}$ or $>150 \mathrm{mg} / 24 \mathrm{~h}$, respectively); cardiomyopathy was defined as the

\section{Predictors of Fabry Cardiomyopathy and Nephropathy}

Multivariate confidence ellipses in the PLS score plots showed that clinical and laboratory variables could differentiate patients with combined cardiomyopathy and nephropathy from those without any of these comorbidities $(p<0.05)$ (Fig. 5b) but could not separate 4 different groups of patients (cardiomyopathy, nephropathy, both, or neither) (Fig. 5a). Then, a PLS analysis was used to discriminate patients with combined cardiomyopathy and nephropathy from those without any of these comorbidities. Variables with VIP higher than 0.8 , considered moderately or highly influential to the separation of presence of ECG abnormalities, LVH, or IVSH. ${ }^{*} p=0.03$ : with versus without cardiomyopathy $(\mathbf{a}) ;{ }^{*} p=0.02$ with versus without cardiomyopathy and nephropathy (c). ECG, electrocardiogram; IVSH, interventricular septal hypertrophy; ELISA, enzyme-linked immunosorbent assay.

groups, were time on ERT, syndecan-1, 3-NT, and GDF15 levels, and systolic and diastolic pressures (Fig. 5c).

\section{Discussion}

This observational study evaluated novel potential biomarkers of organ damage in a cohort of classical Fabry patients on ERT. Our findings suggest that GDF-15 and syndecan-1 may be helpful to identify FD patients with cardiac and kidney damage, while 3-NT may be a marker of cardiac involvement. Moreover, syndecan-1, 3-NT, GDF-15, time on ERT, and arterial pressure were identified as im- 
Fig. 4. Matrix of correlation between time on ERT and novel serum biomarkers. SDC1 , syndecan $-1 .{ }^{*} p=0.04$.
ERT-Time

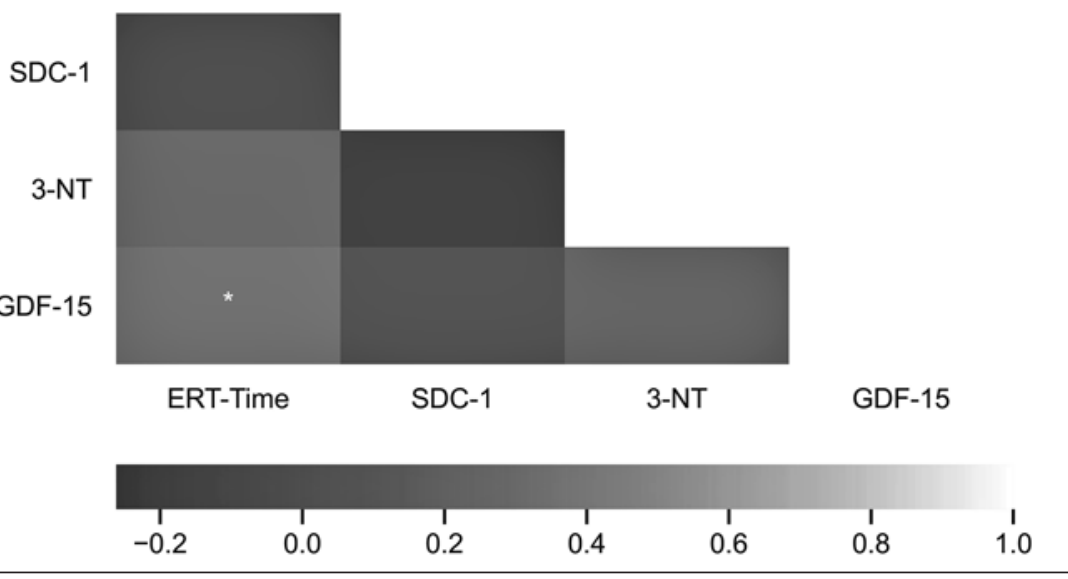

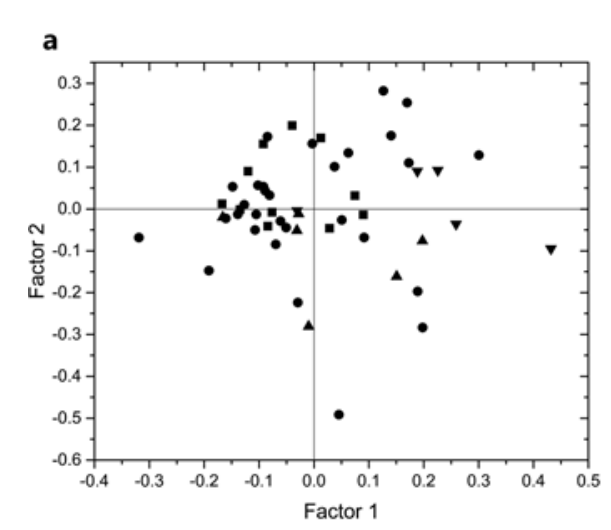

b

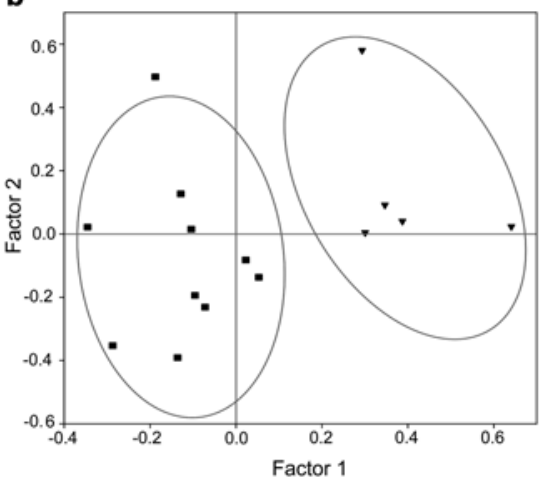

c

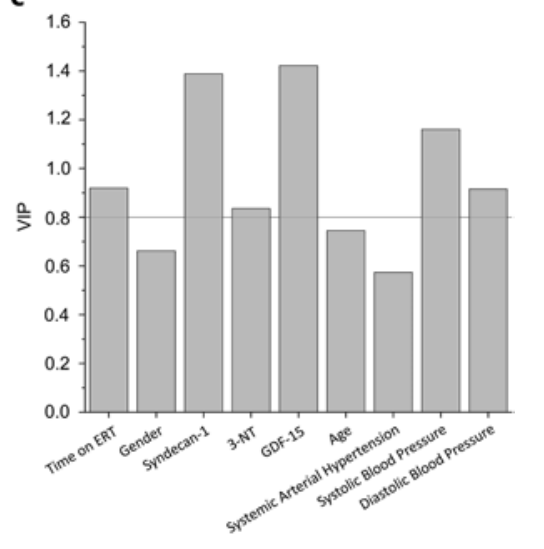

Fig. 5. Differentiation between Fabry patients with or without cardiomyopathy and nephropathy through clinical and laboratory parameters. a PLS among patients with cardiomyopathy (triangle), nephropathy (circle), and both cardiomyopathy and nephropathy (triangle-down), or without any of these comorbidities (square). b PLS between patients with both cardiomyopathy and nephropathy (triangle-down), and without any of these comorbidities (square). c VIP resulting from the PLS; the straight line demonstrates the 0.8 cutoff. Variables with VIP higher than 0.8 were considered moderately or highly influential to the separation of groups. portant variables to differentiate classic Fabry patients with both cardiac and renal involvement from those without these complications. Thus, our results suggest the potential use of these novel biomarkers to identify classic FD patients on ERT with cardiac and renal damages.

Serum 3-NT is a biomarker of oxidative damage induced by nitrogen-free radical species. In our study, even though serum 3-NT levels were not correlated with parameters of cardiac and renal lesion, 3-NT might be a possible biomarker for FD, as indicated by the PLS analysis. Experimental studies using mice models of FD have suggested that 3-NT may play a part on the development and be a marker of vas- culopathy in FD [10, 32]. Interestingly, serum 3-NT levels were over six-fold higher in untreated male patients with classical FD compared to aged- and gender-matched controls, and, similar to our findings, 3-NT levels were not associated with eGFR [10]. As, differently from the aforementioned data, the study population of the present study was composed of classic FD patients, the majority of them females, on ERT with stable disease, the lower levels of 3-NT might suggest that ERT, together with adjunctive therapy, as well as gender, might impact the oxidative stress on those patients. However, this hypothesis should be confirmed in prospective studies with sex-matched controls. 
Syndecan-1, a biomarker of endothelial dysfunction and of glycocalyx damage [33], was also evaluated in our cohort of classic FD patients. Serum levels of syndecan-1 were directly correlated with IVST and were higher, although not significant $(p=0.06)$, in classical FD patients with cardiomyopathy than in those without it. Furthermore, syndecan-1 may be a helpful biomarker to identify classic FD patients with cardiac and renal involvement. Syndecan-1 levels may be elevated in ischemic heart disease, acute heart failure (HF), lupus nephritis, CKD, and acute kidney injury after pediatric cardiac surgery [3337]. In patients with chronic HF, syndecan-1 serum levels were associated with cardiac fibrosis as well as with clinical outcomes in patients with HF with preserved ejection fraction [38]. Thus, our findings support the notion that syndecan-1 may be a useful biomarker of cardiac damage and may be of particular interest in FD, a condition commonly associated with cardiac fibrosis. Even though a previous study reported that syndecan-1 levels were associated with acute kidney injury after pediatric cardiac surgery [33], we found no correlation between syndecan-1 and eGFR. The different type of kidney lesion, type of insult, and age of the population may help to explain the discordance between these findings. Moreover, the fact the most of our patients have CKD G1 may have prevented us from finding any correlation between syndecan-1 and eGFR. Otherwise, a direct correlation between serum syndecan-1 levels and proteinuria was observed. In agreement with these results, it was reported that serum syndecan-1 levels were higher in microalbuminuric than in normoalbuminuric diabetic patients or healthy controls [39] and positively correlated with proteinuria in patients with lupus nephritis [37]. One may hypothesize that endothelial glycocalyx lesion might play a role in the pathogenesis of glomerular disease, including Fabry nephropathy. Furthermore, the association between syndecan-1 and both Fabry cardiomyopathy and nephropathy suggests a bidirectional relationship between these complications and a pivotal role of endothelial injury in the multisystemic involvement in FD.

GDF-15 is a member of the transforming growth factor $\beta$ family that is expressed in a variety of cells, including the cardiovascular system, the immune system, and the kidneys, and may be induced in response to injury [40]. Indeed, GDF-15 has been associated with both cardiovascular damage and CKD [39-41]. In our study, serum GDF-15 levels inversely correlated with eGFR and directly with time on ERT and IVST. Indeed, serum levels of GDF-15 were significantly higher in patients with greater renal damage, as demonstrated by dividing the patients according to the CKD stage and to the tertile of eGFR. Additionally, GDF-15 was higher in classical FD patients with cardiomyopathy associated or not with nephropathy, thus, GDF-15 may be a helpful biomarker to identify classic FD patients with cardiac involvement. Several studies have reported that GDF-15 is independently associated with mortality and cardiovascular events in coronary artery disease and HF patients $[41,42]$ as well as with new onset kidney disease and risk of CKD progression [43-45]. Experimental studies have suggested that GDF-15 is not only a biomarker, but that it also modulates renal and cardiac injuries, possibly providing protection from tissue injury. GDF-15 knockout mice displayed increased tubular injury, manifested by glycosuria, polyuria, and interstitial damage [46]. Additionally, cardiac infarct area was larger in GDF-15 knockout mice than in wild-type mice [47]. GDF-15 is thought to play a protective role by inhibiting myocardial hypertrophy and atherosclerosis in the cardiovascular system and fibrosis in the kidneys [48]. Thus, it has been proposed that increased GDF-15 levels under disease conditions may be an adaptive response to counterbalance organ damage.

There is growing evidence that ERT, mainly if started at a younger age and with lower renal involvement, may lessen FD burden, improving the quality of life of patients and reducing severe clinical events [22]. Giving further support to the importance of timely initiation of ERT, patients who initiated ERT at an older age were more prone to have more severe cardiac and renal involvements. Interestingly, GDF-15 levels were significantly higher in these patients, possibly indicating the presence of underlying chronic lesions, which is expected to occur due to the longer untreated period. The present study provides the framework for further prospective studies addressing the modulation of these biomarkers over time and whether they will predict kidney or cardiovascular outcomes.

Our study has some limitations that need to be recognized. First, it was not possible to assess the biochemical profiles of patients at baseline (before the onset of ERT). Moreover, serum lyso-Gb3, a biomarker of FD burden and of response to therapy, could not be measured [49]. Even though we have found an association between syndecan-1 and GDF-15 in one hand and cardiac and renal involvement in the other, we cannot determine causality. Furthermore, the study was carried out without a control group of healthy subjects or a control-matched population, not Fabry. As so, the results may not be considered specific to FD. We have no data on cardiac imaging magnetic resonance, which prevented us from investigating the association of theses biomarkers, particularly syn- 
decan-1, with cardiac fibrosis. At last, the cross-sectional design of the study prevented us from investigating whether these biomarkers predict outcomes. Even though our findings are promising, we recognize they need to be reproduced in longitudinal studies with a higher number of patients before recommending its use in clinical practice. Importantly, as the serum levels of GDF-15 and syndecan-1 may be measured by enzyme-linked immunosorbent assay, an assay widely used in clinical laboratories, once their utility proved, their incorporation in clinical practice may potentially be easy and affordable.

\section{Conclusions}

Our study is the first to measure GDF-15 and syndecan-1 levels in patients with FD. Our findings suggest that these novel biomarkers are associated with evidence of heart and kidney injury in classical FD patients on ERT. Their potential association with residual risk while on therapy and disease outcomes deserves to be investigated and the present results provide a framework for such research.

\section{Acknowledgments}

The authors would like to express their gratitude to Dr. Mariza Rosa for her invaluable help with sample collection.

\section{Statement of Ethics}

This study protocol was approved by the Ethics Committee of Universidade Federal do Paraná (Curitiba, Brazil) under registration number CEP/UFPR n 2.209.616. All patients provided their written informed consent.

\section{Conflict of Interest Statement}

A.O. is consultant for Sanofi Genzyme and Freeline and has received speaker fees from Amicus; M.D.S.N. has received speaker fees from Sanofi Genzyme. The other authors have no conflicts of interest to declare.

\section{Funding Sources}

P.C.G., R.S.C., and E.M.S. scholarships from Coordenação de Aperfeiçoamento de Pessoal de Nível Superior (CAPES) (Finance Code 001). P.C.G. also received a sandwich scholarship from CAPES. J.B. received scholarship from Universidade Federal do Paraná/Tesouro Nacional. A.O. and M.D.S.N. were funded by Programa Ramón y Cajal, FRIAT, ISCIII RETIC REDINREN RD 016/009 and ISCIII FIS/Fondos FEDER (PI18/01366, PI19/00588, PI19/00815), Sociedad Española de Nefrología.

\section{Author Contributions}

P.C.G., G.B., R.S.C., B.B., J.B., and R.H. performed the experiments and collected the data; P.C.G., E.M.S., and R.S.C. performed the statistical analysis; A.E.M.S., F.C.B., A.O., M.D.S.N., and A.M.M. provided advice on the experiments and supported the interpretation of results; P.C.G, A.E.M.S., and F.C.B. were responsible for the study design, drafted, and reviewed the manuscript. All authors read and approved the content of the manuscript.

\section{Data Availability Statement}

All data generated or analyzed during this study are included in this article. Further inquiries can be directed to the corresponding author.

\section{References}

1 Desnick RJ. Fabry disease. Rosenberg's Molecular Genetics basis neurology psychiatry disease: Elsevier; 2015. p. 419-30.

2 Germain DP. Fabry disease. Orphanet J Rare Dis. 2010;5:30.

3 Arends M, Hollak C, Biegstraaten M. Quality of life in patients with Fabry disease: a systematic review of the literature. Orphanet J Rare Dis. 2015; 10:77.

4 Linhart A, Kampmann C, Zamorano JL, Sunder-Plassmann G, Beck M, Mehta A, et al. Cardiac manifestations of Anderson-Fabry disease: results from the international Fabry outcome survey. Eur Heart J. 2007;28:122835.

GDF-15 and Syndecan-1 in Fabry Disease under ERT
5 Desnick RJ. Fabry disease, an under-recognized multisystemic disorder: expert recommendations for diagnosis, management, and enzyme replacement therapy. Ann Intern Med. 2003;138:338.

6 MacDermot KD. Anderson-fabry disease: clinical manifestations and impact of disease in a cohort of 60 obligate carrier females. J Med Genet. 2001;38:769-75.

7 Veloso VSP, Ataides TL, Canziani MEF, Veloso MP, da Silva NA, Barreto DV, et al. A novel missense GLA mutation (p.G35V) detected in hemodialysis screening leads to severe systemic manifestations of fabry disease in men and women. Nephron. 2018;138:14756.
8 Park JL, Whitesall SE, D'Alecy LG, Shu L, Shayman JA. The vascular dysfunction in the a-galactosidase A knockout mouse is an endothelial cell, plasma membrane-based defect. Clin Exp Pharmacol Physiol. 2008;35: 1156-63.

9 Kang JJE, Shu L, Park JL, Shayman JA, Bodary PF. Endothelial nitric oxide synthase uncoupling and microvascular dysfunction in the mesentery of mice deficient in $\alpha$-galactosidase A. Am J Physiol Gastrointest Liver Physiol. 2014;306:G140-6. 
10 Shu L, Vivekanandan-Giri A, Pennathur S, Smid BE, Aerts JMFG, Hollak CEM, et al. Establishing 3-nitrotyrosine as a biomarker for the vasculopathy of Fabry disease. Kidney Int. 2014;86:58-66.

11 Shen JS, Meng XL, Moore DF, Quirk JM, Shayman JA, Schiffmann R, et al. Globotriaosylceramide induces oxidative stress and upregulates cell adhesion molecule expression in Fabry disease endothelial cells. Mol Genet Metab. 2008;95:163-8.

12 Rozenfeld P, Feriozzi S. Contribution of inflammatory pathways to Fabry disease pathogenesis. Mol Genet Metab. 2017;122:19-27.

13 Sanchez-Niño MD, Carpio D, Sanz AB, RuizOrtega M, Mezzano S, Ortiz A. Lyso-Gb3 activates Notch1 in human podocytes. Hum Mol Genet. 2015;24:5720-32.

14 Eng CM, Guffon N, Wilcox WR, Germain DP, Lee P, Waldek S, et al. Safety and efficacy of recombinant human a-galactosidase a replacement therapy in fabry's disease. N Engl J Med. 2001;345:9-16.

15 Warnock DG. Enzyme replacement therapy and fabry kidney disease: quo vadis? J Am Soc Nephrol. 2007;18:1368-70.

16 Thurberg BL, Fallon JT, Mitchell R, Aretz T, Gordon RE, O'Callaghan MW. Cardiac microvascular pathology in fabry disease. Circulation. 2009;119:2561-7.

17 Germain DP, Charrow J, Desnick RJ, Guffon N, Kempf J, Lachmann RH, et al. Ten-year outcome of enzyme replacement therapy with agalsidase beta in patients with Fabry disease. J Med Genet. 2015:353-8.

18 Germain DP, Weidemann F, Abiose A, Patel MR, Cizmarik M, Cole JA, et al. Analysis of left ventricular mass in untreated men and in men treated with agalsidase- $\beta$ : data from the Fabry Registry. Genet Med. 2013;15:958-65.

19 Fellgiebel A, Gartenschläger M, Wildberger K, Scheurich A, Desnick RJ, Sims K. Enzyme replacement therapy stabilized white matter lesion progression in Fabry disease. Cerebrovasc Dis. 2014;38:448-56.

20 El Dib R, Gomaa H, Ortiz A, Politei J, Kapoor A, Barreto F. Enzyme replacement therapy for Anderson-Fabry disease: a complementary overview of a Cochrane publication through a linear regression and a pooled analysis of proportions from cohort studies. PLoS One. 2017;12:e0173358.

21 Warnock DG, Ortiz A, Mauer M, Linthorst GE, Oliveira JP, Serra AL, et al. Renal outcomes of agalsidase beta treatment for Fabry disease: role of proteinuria and timing of treatment initiation. Nephrol Dial Transplant. 2012;27:1042-9.

22 Ortiz A, Abiose A, Bichet DG, Cabrera G, Charrow J, Germain DP, et al. Time to treatment benefit for adult patients with Fabry disease receiving agalsidase $\beta$ : data from the Fabry registry. J Med Genet. 2016;53:495-502.

23 Hopkin RJ, Cabrera G, Charrow J, Lemay R, Martins AM, Mauer M, et al. Risk factors for severe clinical events in male and female pa- tients with Fabry disease treated with agalsidase beta enzyme replacement therapy: data from the Fabry registry. Mol Genet Metab. 2016;119:151-9.

24 Seydelmann N, Liu D, Krämer J, Drechsler C, Hu K, Nordbeck P, et al. High-sensitivity troponin: a clinical blood biomarker for staging cardiomyopathy in fabry disease. J Am Heart Assoc. 2016;5:13-6.

25 Ortiz A, Germain DP, Desnick RJ, Politei J, Mauer M, Burlina A, et al. Fabry disease revisited: management and treatment recommendations for adult patients. Mol Genet Metab. 2018;123:416-27.

26 Levey AS, Stevens LA, Schmid CH, Zhang YL, Castro AF, Feldman HI, et al. A new equation to estimate glomerular filtration rate. Ann Intern Med. 2009;150:604-12.

27 Glassock RJ, Winearls C. Ageing and the glomerular filtration rate: truths and consequences. Trans Am Clin Climatol Assoc. 2009; 120:419-28.

28 Riccio E, Sabbatini M, Bruzzese D, Annicchiarico Petruzzelli L, Pellegrino A, Spinelli L, et al. Glomerular hyperfiltration: an early marker of nephropathy in fabry disease. Nephron. 2019;141:10-7.

29 Kidney Disease Improving Global Outcomes. Abstract. Kidney Int Suppl. 2013;3:4.

30 Ortiz A, Oliveira JP, Waldek S, Warnock DG, Cianciaruso B, Wanner C. Nephropathy in males and females with Fabry disease: crosssectional description of patients before treatment with enzyme replacement therapy. Nephrol Dial Transplant. 2008;23:1600-7.

31 Ortiz A, Abiose A, Bichet DG, Cabrera G, Charrow J, Germain DP, et al. Time to treatment benefit for adult patients with Fabry disease receiving agalsidase $\beta$ : data from the Fabry registry. J Med Genet. 2016;53:495-502.

32 Bodary PF, Shen Y, Vargas FB, Bi X, Ostenso $\mathrm{KA}, \mathrm{Gu} \mathrm{S}$, et al. a-galactosidase A deficiency accelerates atherosclerosis in mice with apolipoprotein E deficiency. Circulation. 2005; 111:629-32.

33 Nieuwdorp M, Meuwese MC, Vink H, Hoekstra JB, Kastelein JJ, Stroes ES. The endothelial glycocalyx: a potential barrier between health and vascular disease. Curr Opin Lipidol. 2005; 16:507-11.

34 Cavalcante CT, Castelo Branco KM, Pinto Júnior VC, Meneses GC, de Oliveira Neves FM, de Souza NMG, et al. Syndecan-1 improves severe acute kidney injury prediction after pediatric cardiac surgery. J Thorac Cardiovasc Surg. 2016;152:178-86.e2.

35 Demissei BG, Valente MAE, Cleland JG, O'Connor CM, Metra M, Ponikowski P, et al. Optimizing clinical use of biomarkers in high-risk acute heart failure patients. Eur J Heart Fail. 2016;18:269-80.

36 Padberg JS, Wiesinger A, di Marco GS, Reuter S, Grabner A, Kentrup D, et al. Damage of the endothelial glycocalyx in chronic kidney disease. Atherosclerosis. 2014;234:33543.
37 Kim KJ, Kim JY, Baek IW, Kim WU, Cho CS. Elevated serum levels of syndecan-1 are associated with renal involvement in patients with systemic lupus erythematosus. J Rheumatol. 2015;42:202-9.

38 Tromp J, Van Der Pol A, Klip IT, De Boer RA Jaarsma T, Van Gilst WH, et al. Fibrosis marker syndecan-1 and outcome in patients with heart failure with reduced and preserved ejection fraction. Circ Hear Fail. 2014;7:457-62.

39 Svennevig K, Kolset SO, Bangstad HJ. Increased syndecan- 1 in serum is related to early nephropathy in type 1 diabetes mellitus patients. Diabetologia. 2006;49:2214-6.

40 Preusch MR, Baeuerle M, Albrecht C, Blessing E, Bischof M, Katus HA, et al. GDF-15 protects from macrophage accumulation in a mousemodel of advanced atherosclerosis. Eur J Med Res. 2013;18:1-6.

41 Rohatgi A, Patel P, Das SR, Ayers CR, Khera A, Martinez-Rumayor A, et al. Association of growth differentiation factor-15 with coronary atherosclerosis and mortality in a young, multiethnic population: observations from the dallas heart study. Clin Chem. 2012;58: 172-82.

42 Anand IS, Kempf T, Rector TS, Tapken H, Allhoff T, Jantzen F, et al. Serial measurement of growth-differentiation factor-15 in heart failure. Circulation. 2010;122:1387-95.

43 Nair V, Robinson-Cohen C, Smith MR, Bellovich KA, Bhat ZY, Bobadilla $M$, et al. Growth differentiation factor-15 and risk of CKD progression. J Am Soc Nephrol. 2017; 28:2233-40.

44 Lajer M, Jorsal A, Tarnow L, Parving HH, Rossing P. Plasma growth differentiation factor-15 independently predicts all-cause and cardiovascular mortality as well as deterioration of kidney function in type 1 diabetic patients with nephropathy. Diabetes Care. 2010; 33:1567-72.

45 Ho JE, Hwang SJ, Wollert KC, Larson MG, Cheng S, Kempf T, et al. Biomarkers of cardiovascular stress and incident chronic kidney disease. Clin Chem. 2013;59:1613-20.

46 Mazagova M, Buikema H, van Buiten A, Duin M, Goris M, Sandovici M, et al. Genetic deletion of growth differentiation factor 15 augments renal damage in both type 1 and type 2 models of diabetes. Am J Physiol Physiol. 2013;305:F1249-64.

47 Kempf T, von Haehling S, Peter T, Allhoff T, Cicoira M, Doehner W, et al. Prognostic utility of growth differentiation factor- 15 in patients with chronic heart failure. J Am Coll Cardiol. 2007;50:1054-60.

48 Emmerson PJ, Duffin KL, Chintharlapalli S, $\mathrm{Wu}$ X. GDF15 and growth control. Front Physiol. 2018;9:1-7.

49 Rombach SM, Dekker N, Bouwman MG, Linthorst GE, Zwinderman AH, Wijburg FA, et al. Plasma globotriaosylsphingosine: Diagnostic value and relation to clinical manifestations of Fabry disease. Biochim Biophys Act. 2010;1802:741-8. 